\title{
CRITIC-Entropy based Fuzzy Decision Making Models: A Systematic Analysis
}

Aldring $\sqrt{1}$ and Ajay $\mathrm{D}^{*, 2}$

Received: 29 April 2021/ Accepted: 31 May 2021/ Published online: 18 June 2021

(C)Sacred Heart Research Publications 2017

\begin{abstract}
This research article presents a comprehensive analysis of weighting methods used in fuzzy multi attribute decision making (MADM) methods. These methods involve various criteria in order to evaluate alternatives and determining the weights of criteria is a significant problem that arises very often in many MADM problems. In this research paper, CRTITIC and Entropy weighting methods have been used for finding criteria's weights like in many research works. Using these unsupervised methods of assigning criteria weights, seven fuzzy MADM methods are examined in the context of ranking the best company to invest in. From the results of these methods, ranking order of alternatives is obtained and are analysed for reliability.
\end{abstract}

Key words: Fuzzy sets, MADM, CRITIC, Entory, Decision Models

AMS classification: 03E72, 03B52, 94D05

\section{Introduction}

In some of the situations characterized by uncertainty and imprecision, Boolean logic fails to represent adequately. So in order to handle these uncertain situations, the fuzzy set theory [15] has been extended to different forms like Intuitionistic fuzzy sets (IFSs) [1], Pythagorean fuzzy sets (PFSs) [14], Neutrosophic sets (NSs)[10], Spherical fuzzy sets (SFSs) [8] and so on. It is evident from the literature that these concepts have many real time applications in multi attribute decision making (MADM) [5, 6] methods. These MADM methods are widely used in decision making theory. They involve several decision factors or criteria to deal with in any decision making situation. At same time, the attribute weights are extremely important in decision-making process. Decision maker is always struggling to assign criteria

\footnotetext{
1,2 Department of Mathematics, Sacred Heart College (Autonomous), Tirupattur - 635601, Tamilnadu, India. Email: 1jaldring24@gmail.com, ${ }^{*, 2}$ dajaypravin@gmail.com
} 
weights in selection problem. For this purpose, two unsupervised weighting methods, namely CRITIC [7] and Entropy [11], are widely used, and some of well known fuzzy decision making methods were utilized based on attribute weighting methods. Many researchers have undertaken such analysis and they differ in their selective approach. For example, CRITIC and Entropy based fuzzy TOPSIS methods have been implemented in supply chain risk assessment [4] and petrochemical industries [12]. Also, Many MADM methods have been developed based on entropy measure [3, 9]. Moreover, Sahin has done a comprehensive analysis of six weighting and seven multi criteria methods [13].

The rest of this paper is organized as follows: Section 2 describes the preliminaries; Section 3 gives the methodology of weighting methods; Section 4 presents the combined fuzzy MCDM methods with a case study in order to test the applicability of the decision making tools. The comparative analysis is given in section 5. Finally section 6 gives conclusion.

\section{Preliminaries}

Definition 2.1 [15] A fuzzy set $\tilde{\mathbb{F}}$ is defined on a universe of discourse $\Re$ as the form:

$$
\tilde{\mathbb{F}}=\left\{\left\langle\xi_{\tilde{\mathbb{F}}}(\dot{r})\right\rangle \mid \dot{r} \in \Re\right\},
$$

where $\xi_{\tilde{\mathbb{F}}}(\dot{r}): \Re \rightarrow[0,1]$. Here $\xi_{\tilde{\mathbb{F}}}(\dot{r})$ denotes membership function to each $\dot{r}$.

Definition 2.2 [1] An intuitionistic fuzzy sets $\tilde{\mathbb{I}_{\mathbb{F}}}$ is defined as a set of ordered pairs over a universal set $\Re$ given by

$$
\tilde{\mathbb{I}_{\mathbb{F}}}=\left\{\left\langle\left(\xi_{\tilde{\mathbb{I}_{\mathbb{F}}}}(\dot{r}), \psi_{\tilde{\mathbb{I}}_{\mathbb{F}}}(\dot{r})\right)\right\rangle \mid \dot{r} \in \Re\right\},
$$

where $\xi_{\tilde{\mathbb{I}_{F}}}(\dot{r}): \Re \rightarrow[0,1], \psi_{\tilde{\mathbb{I}_{\mathbb{F}}}}(\dot{r}): \Re \rightarrow[0,1]$ and with the condition $\xi_{\tilde{\mathbb{I}_{F}}}(\dot{r})+\psi_{\tilde{\mathbb{I}_{\mathbb{F}}}}(\dot{r}) \leq 1$ for each element $\dot{r} \in \Re$. Here the membership and non-membership functions are denoted as $\xi_{\tilde{\mathbb{I}}_{\mathbb{F}}}(\dot{r})$ and $\psi_{\tilde{\mathbb{I}}_{\mathbb{F}}}(\dot{r})$ respectively. Suppose that the condition $\xi_{\tilde{\mathbb{I}}_{\mathbb{F}}}^{2}(\dot{r})+\psi_{\tilde{\mathbb{I}}_{\mathbb{F}}}^{2}(\dot{r}) \leq$ 1 satisfies for each element $\dot{r} \in \Re$, then it is called Pythagorean fuzzy sets [14].

\section{Weighting methods}

(i). CRITIC Method: The CRitria Importance Thorough Inter critria Correlation (CRITIC) method [7] is used to find criteria weight based on the contrast intensity and conflict evaluation of the decision problem. The steps of CRITIC method are given to determine the attribute weight under fuzzy decision matrix. 
ISSN: 2456-8686, 5(1), 2021:132-141

https://doi.org/10.26524/cm100

Step 1. The decision matrix $[D M] \forall i=1,2,3, \ldots, m, j=1,2,3, \ldots, n$ is formed:

$$
[D M]=\left[\lambda_{i j}\right]_{m \times n}=\left[\begin{array}{cccc}
\lambda_{11} & \lambda_{12} & \ldots & \lambda_{1 m} \\
\lambda_{21} & \lambda_{22} & \ddots & \lambda_{2 m} \\
\vdots & \vdots & \ddots & \vdots \\
\lambda_{n 1} & \lambda_{n 2} & \cdots & \lambda_{n m}
\end{array}\right]
$$

Step 2. Decision matrix is normalized using the following equation

$$
\left[D M^{*}\right]=\left[\lambda_{i j}^{*}\right]_{m \times n}=\frac{\lambda_{i j}-\min _{i} \lambda_{i j}}{\max _{i} \lambda_{i j}-\min _{i} \lambda_{i j}}
$$

Step 3. Calculate the correlation coefficient of the attribute $\mathcal{C}_{j}$ to $\mathcal{C}_{k}$ by Eq.2

$$
\eta_{j k}=\frac{\sum_{i=1}^{m}\left(\lambda_{i j}^{*}-\overline{\lambda_{j}^{*}}\right) \cdot\left(\lambda_{i k}^{*}-\overline{\lambda_{k}^{*}}\right)}{\sqrt{\sum_{i=1}^{m}\left(\lambda_{i j}^{*}-\overline{\lambda_{j}^{*}}\right)^{2} \cdot \sum_{i=1}^{m}\left(\lambda_{i k}^{*}-\overline{\lambda_{k}^{*}}\right)^{2}}}
$$

where $\overline{\lambda_{j}^{*}}=\frac{1}{m} \cdot \sum_{i=1}^{m} \lambda_{i j}^{*}$ and $\overline{\lambda_{k}^{*}}=\frac{1}{m} \cdot \sum_{i=1}^{m} \lambda_{i k}^{*}$

Step 4. Estimate the standard deviation for each attribute

$$
\nu_{j}=\sqrt{\frac{1}{m} \sum_{i=1}^{m}\left(\lambda_{i j}^{*}-\overline{\lambda_{j}^{*}}\right)^{2}}, \forall j=1,2,3, \ldots, n
$$

Step 5. Compute the deviation degree $\theta$ of criterion $\mathcal{C}_{j}$ from the other criteria

$$
\theta_{j}=\nu_{j} \cdot \sum_{k=1}^{n}\left(1-\eta_{j k}\right), j=1,2,3, \ldots, n
$$

Step 6. Obtain the original weights of attributes

$$
\varpi_{j}=\frac{\theta_{j}}{\sum_{j=1}^{n} \theta_{j}} \quad \forall j=1,2,3, \ldots, n
$$


ISSN: 2456-8686, 5(1), 2021:132-141

https://doi.org/10.26524/cm100

(ii). Entropy Method: The entropy concept, which is a measure of uncertainty in information expressed in terms of probability theory, was introduced by Shannon [11. Shannon's entropy method interprets the relative intensities of the criterion importance depending on the discrimination among data to evaluate the relative weights. These are the steps involved in the Shannon's entropy method:

Step 1. Normalize the initial decision matrix

$$
\left[D M^{*}\right]=\left[\lambda_{i j}^{*}\right]_{m \times n}=\frac{\lambda_{i j}}{\sum_{i=1}^{m} \lambda_{i j}^{2}} \forall i=1,2,3, \ldots, m, j=1,2,3, \ldots, n
$$

Step 2. Calculate the entropy of each criterion

$$
E_{j}=-K \sum_{i=1}^{m} \lambda_{i j}^{*} \ln \lambda_{i j}^{*}, \quad K=\frac{1}{\ln m}
$$

Step 3. Calculate the variation coefficient of criterion $\mathcal{C}_{j}$

$$
\Theta_{j}=\left|1-E_{j}\right|
$$

Step 4. Determine the weight of each criterion $\mathcal{C}_{j}$ by the following equation

$$
W_{j}=\frac{\Theta_{j}}{\sum_{i=1}^{m} \Theta_{j}}
$$

\section{MCDM methods}

In this section, the CRITIC and Entropy weighting methods are used to analyze seven fuzzy decision making methods such as weighted sum method (WSM), weighted product method (WPM), the multiple criteria optimization compromise solution (VIKOR), the technique for order performance by similarity to the ideal solution (TOPSIS), the Weighted Aggregates Sum Product Assessment (WASPAS), COmbinative Distance-Based ASsessment (CODAS), and the Evaluation Based on Distance from Average Solution (EDAS). In order to make a comprehensive study the following case study has been taken.

4.1 Case study: An illustrative example on the selection problem of investment alternatives is taken from [2] to analyse the mentioned MCDM methods with fuzzy decision matrix. A company wants a sum of money to be invested in an industry. 
Then the committee suggests the following four feasible alternatives: $\mathcal{A}_{1}$-is a textile company; $\mathcal{A}_{2}$-is an automobile company; $\mathcal{A}_{3}$-is a computer company; $\mathcal{A}_{4}$-is a software company. Suppose that three attributes namely, $\mathcal{C}_{1}$-is the risk; $\mathcal{C}_{2}$-is the growth; $\mathcal{C}_{3}$-is the environmental impact; are taken into the evaluation requirements of the alternatives. Then the expert or decision maker is asked to evaluate each alternative on attributes in the form of fuzzy numbers. Thus, the assessment data can be represented by fuzzy decision matrix $D=\left[\lambda_{i j}\right]_{4 \times 3}$ and it is given by

$$
\begin{aligned}
& \begin{array}{ccc}
\mathcal{C}_{1} & \mathcal{C}_{2} & \mathcal{C}_{3}
\end{array} \\
& \left.[D M]=\left[\lambda_{i j}\right]_{4 \times 3}=\quad \begin{array}{ccccc} 
& \mathcal{A}_{1} & 0.5 & 0.6 & 0.4 \\
& \mathcal{A}_{2} & 0.7 & 0.8 & 0.9 \\
& \mathcal{A}_{3} & 0.4 & 0.7 & 0.6 \\
& \mathcal{A}_{4} & 0.3 & 0.8 & 0.5
\end{array}\right)
\end{aligned}
$$

\section{Weighting criteria values:}

(i). Based on the CRITIC method, the criteria weights are 0.3684, 0.4393, 0.1923.

(ii). Similarly for Entropy method, the criteria weights are 0.4725, 0.0663, 0.4612. However, the objective weighting method (CRITIC and Entropy) results are not identical. The results of CRITIC method indicates 'growth' is important factor and Entropy method indicates 'risk' is the critical factor.

\subsection{Fuzzy models using CRITIC weights:}

1. Algorithm: WSM $=\max _{i} \sum_{j=1}^{n} \lambda_{i j} \varpi_{j}$ for $i=1,2,3, \ldots, m$. $\varpi_{j}$ is the weight of the $j^{\text {th }}$ attribute, and $\lambda_{i j}$ is the corresponding value. Then, the preference score of WSM: $\mathcal{A}_{1}=0.67808, \mathcal{A}_{2}=1, \mathcal{A}_{3}=0.7231, \mathcal{A}_{4}=0.70402$

2. Algorithm: WPM $=\prod_{j=1}^{n}\left(\lambda_{i j}\right)^{\varpi_{j}}$ for $i=1,2,3, \ldots, m$. $\varpi_{j}$ is the weight of the $j^{\text {th }}$ attribute, and $\lambda_{i j}$ is the corresponding value. Then, the preference score of WPM: $\mathcal{A}_{1}=0.6661, \mathcal{A}_{2}=1, \mathcal{A}_{3}=0.7098, \mathcal{A}_{4}=0.6537$

3. Algorithm: WASPAS $=\lambda * W S M+(1-\lambda) * W P M$ when $\lambda=0.5$. Then, joint generalized preference score: $\mathcal{A}_{1}=0.6721, \mathcal{A}_{2}=1, \mathcal{A}_{3}=0.7164, \mathcal{A}_{4}=0.6788$

4. Algorithm: TOPSIS 
ISSN: 2456-8686, 5(1), 2021:132-141

https://doi.org/10.26524/cm100

(i). Calculate the normalized matrix

(ii). Calculate the weighted mormalized matrix

(iii). Calculate the positive and negative best

(iv). Find euclidean distance from Ideal Best and Worst

(v). Calculate the performance score

After executing TOPSIS method based on CRITIC method, the final performance score values: $\mathcal{A}_{1}=0.3771, \mathcal{A}_{2}=1, \mathcal{A}_{3}=0.3139, \mathcal{A}_{4}=0.2793$

5. Algorithm: VIKOR

(i). Find the $f^{*}$ and $f^{-}$indexes

(ii). Determine the $S$ and $R$ indexes

(iii). Calculate the VIKOR indexes

(iv). Find the compromise solution and analyze the results

Then, the compromise solution : $\mathcal{A}_{1}=\mathrm{NaN}, \mathcal{A}_{2}=0, \mathcal{A}_{3}=0.6892, \mathcal{A}_{4}=0.7394$

6. Algorithm: EDAS

(i). Determine the average solution

(ii). Calculate the positive Distance from Average (PDA)and negative Distance from Average (NDA)

(iii). Calculate SP(weighted Sum of PDA), SN(weighted Sum of NDA), NSP, NSN

(iv). Calculate the appraisal score solution

Then, the appraisal score solution : $\mathcal{A}_{1}=0.0519, \mathcal{A}_{2}=1, \mathcal{A}_{3}=0.3202, \mathcal{A}_{4}=0.0480$

7. Algorithm: CODAS

(i). Calculate the normalized matrix

(ii). Calculate the weighted normalized matrix

(iii). Calculate the negative idle solution

(iv). Calculate the Euclidean distance from the negative solution

(v). Calculate the Taxicab distance from the negative solution

(vi). Calculate Relative assessment matrix for $\tau=0.01$

(vii). Calculate the assessment score 
ISSN: 2456-8686, 5(1), 2021:132-141

https://doi.org/10.26524/cm100

Then, the final values : $\mathcal{A}_{1}=-0.0946, \mathcal{A}_{2}=0.6185, \mathcal{A}_{3}=-0.1691, \mathcal{A}_{4}=-0.0737$

\subsection{Fuzzy models using Entropy weights:}

Similarly the mentioned methods have been executed based on Entropy weighting method and the final results are calculated and shown in the following table.

\begin{tabular}{ccccc}
\hline Methods & $\mathcal{A}_{1}$ & $\mathcal{A}_{2}$ & $\mathcal{A}_{3}$ & $\mathcal{A}_{4}$ \\
\hline \hline En-WSM & 0.5922 & 1 & 0.6355 & 0.5250 \\
\hline En-WPM & 0.5758 & 1 & 0.6311 & 0.5110 \\
\hline En-WASPAS & 0.5840 & 1 & 0.6333 & 0.518 \\
\hline En-TOPSIS & 0.3147 & 1 & 0.3271 & 0.1361 \\
\hline En-VIKOR & $\mathrm{NaN}$ & 0 & 0.8190 & 1.0000 \\
\hline En-EDAS & 0.0519 & 1 & 0.3202 & 0.0480 \\
\hline En-CODAS & -0.0426 & 1.1452 & -0.0944 & -0.3045 \\
\hline
\end{tabular}

Based on the finding results, we observe that $\mathcal{A}_{2}$ is the best alternative in overall.

\section{Comparative Analysis}

To compare the results of this study, a pictorial representation is given in Figures. 1 and 2 for the better understanding.

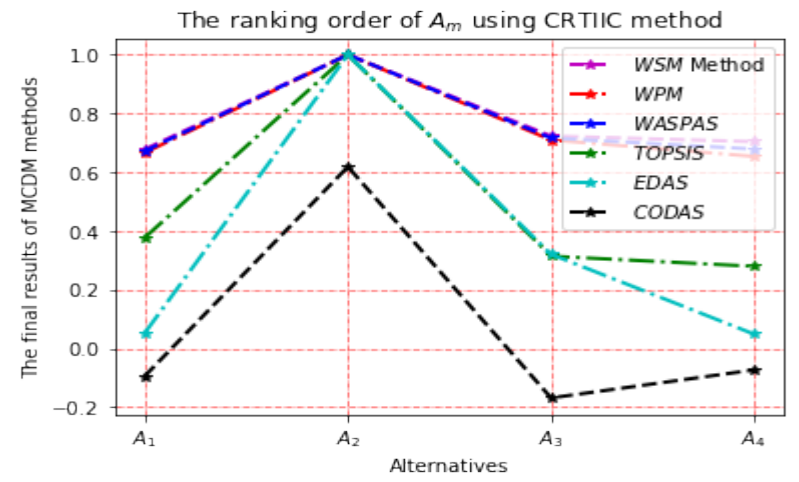

Figure 1: The final result of MCDM methods using CRITIC weighting method 
ISSN: 2456-8686, 5(1), 2021:132-141

https://doi.org/10.26524/cm100

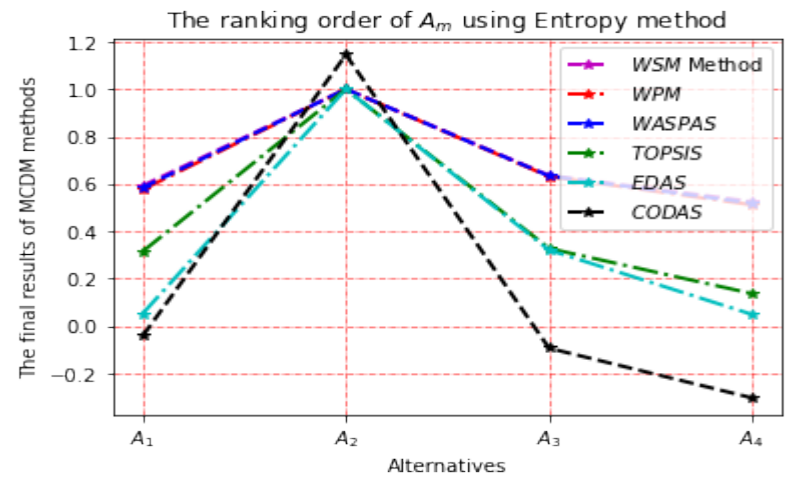

Figure 2: The final result of MCDM methods using Entropy weighting method

\begin{tabular}{c|c|c}
\hline MCDM Methods & Ranking order : CRITIC & Ranking order : Entropy \\
\hline \hline WSM & $A_{2} \succ A_{3} \succ A_{1} \succ A_{4}$ & $A_{2} \succ A_{3} \succ A_{1} \succ A_{4}$ \\
\hline WPM & $A_{2} \succ A_{3} \succ A_{1} \succ A_{4}$ & $A_{2} \succ A_{3} \succ A_{1} \succ A_{4}$ \\
\hline WASPAS & $A_{2} \succ A_{3} \succ A_{1} \succ A_{4}$ & $A_{2} \succ A_{3} \succ A_{1} \succ A_{4}$ \\
\hline TOPSIS & $A_{2} \succ A_{3} \succ A_{1} \succ A_{4}$ & $A_{2} \succ A_{3} \succ A_{1} \succ A_{4}$ \\
\hline EDAS & $A_{2} \succ A_{3} \succ A_{1} \succ A_{4}$ & $A_{2} \succ A_{3} \succ A_{1} \succ A_{4}$ \\
\hline CODAS & $A_{2} \succ A_{3} \succ A_{1} \succ A_{4}$ & $A_{2} \succ A_{3} \succ A_{1} \succ A_{4}$ \\
\hline
\end{tabular}

From above table, we observe that the ranking order of the alternatives are the same for all fuzzy decision making methods. Hence we find that the objective weighting methods such as CRITIC and Entropy are efficient and more reliable to deal with all types of fuzzy MCDM methods.

\section{Conclusion}

In this study, two objective weighting methods and seven fuzzy MCDM methods have been used for comparative analysis of ranking of alternatives. The weighting vectors are involved in fuzzy decision making methods and they play a very important role in finding the best output. According to the evaluation and results of the methods, we conclude that the seven methods under analysis are more reliable in decision making as the ranking obtained are almost similar. This research could be 
ISSN: 2456-8686, 5(1), 2021:132-141

https://doi.org/10.26524/cm100

extended for the systematic analysis of decision making methods based on complex fuzzy sets.

\section{References}

[1] Atanassov, K.T., Intuitionistic fuzzy sets. Fuzzy Sets Syst. 20(1), 8796 (1986).

[2] Ajay D, Said Broumi, \& Aldring J, An MCDM Method under Neutrosophic Cubic Fuzzy Sets with Geometric Bonferroni Mean Operator. Neutrosophic Sets and Systems, 32, 187-202 (2020), Retrieved from http://fs.unm.edu/NSS2/index.php/111/article/view/326

[3] Ajay D, \& Aldring J, A Decision Making Technique Based on Similarity Measure and Entropy of Bipolar Neutrosophic Sets. The International journal of analytical and experimental modal analysis, 11(9), 520-529 (2019).

[4] Abdel-Basset M, \& Mohamed R, A novel plithogenic TOPSIS-CRITIC model for sustainable supply chain risk management. Journal of Cleaner Production, 247, 119586 (2020).

[5] Ajay D, Aldring A, \& Nivetha S, Neutrosophic Cubic Fuzzy Dombi Hamy Mean Operators with Application to Multi-Criteria Decision Making. Neutrosophic Sets and Systems, 38, 293-316, (2020), Retrieved from http://fs.unm.edu/NSS2/index.php/111/article/view/925

[6] Borg SJ, Ajay D, Aldring J, Exponential operational laws of pythagorean fuzzy projection models for decision making, Advances in Mathematics: Scientific Journal, 9(8), 61076114, (2020), DOI: https://doi.org/10.37418/amsj.9.8.78

[7] Diakoulaki D, Mavrotas G, Papayannakis L, Determining objective weights in multiple criteria problems: the CRITIC method. Comput OR, 22, 763-770 (1995).

[8] Gundogdu FK, Kahraman C, Spherical fuzzy sets and spherical fuzzy TOPSIS method. J. Intell. Fuzzy Syst. 36(1), 337352, (2019). DOI:10.3233/JIFS-181401

[9] Lin M, Huang C, \& Xu Z, TOPSIS method based on correlation coefficient and entropy measure for linguistic pythagorean fuzzy sets and its application to multiple attribute decision making. Complexity, (2019).

[10] Smarandache FA, Unifying Field in Logics, Neutrosophic Logic, Neutrosophy, Neutrosophic Set and Neutrosophic Probabilty. 4th (eds) American Research Press, Rehoboth, DE, USA, 1999. 
ISSN: 2456-8686, 5(1), 2021:132-141

https://doi.org/10.26524/cm100

[11] Shannon CE, A mathematical theory of communication. Bell Syst Tech J 27, 379423 (1948), https://doi.org/10.1002/j.1538-7305.1948.tb01338.x

[12] Salehi V, Zarei H, Shirali GA, \& Hajizadeh K, An entropy-based TOPSIS approach for analyzing and assessing crisis management systems in petrochemical industries. Journal of Loss Prevention in the Process Industries, 67, 104241, (2020).

[13] Sahin M, A comprehensive analysis of weighting and multicriteria methods in the context of sustainable energy. Int. J. Environ. Sci. Technol. 18, 15911616 (2021). https://doi.org/10.1007/s13762-020-02922-7

[14] Yager R, Pythagorean fuzzy subsets. Joint IFSA World Congress and NAFIPS Annual Meeting, Edmonton, Canada, 2013.

[15] Zadeh LA, Fuzzy sets. Inf Control, 8, 338-353 (1965). 